\title{
Tuning constitutive recombinant gene expression in Lactobacillus plantarum
}

\author{
Christopher Tauer, Stefan Heinl', Esther Egger, Silvia Heiss and Reingard Grabherr
}

\begin{abstract}
Background: Lactobacillus plantarum constitutes a well-recognized food-grade system for the expression of recombinant proteins in the field of industrial and medical biotechnology. For applications in vivo or in biotechnological processes, the level of expression of e.g. antigens or enzymes is often critical, as expression levels should be of a certain effectiveness, yet, without putting too much strain to the overall system. The key factors that control gene expression are promoter strength, gene copy number and translation efficiency. In order to estimate the impact of these adjusting screws in $L$. plantarum CD033, we have tested several constitutive promoters in combination with high and low copy number plasmid backbones and varying space between the Shine-Dalgarno sequence and the start-codon.

Results: By combining strong promoters, such as transcription elongation factor promoters, isolated from L. plantarum CD033 and L. buchneri CD034, a synthetic promoter, originally derived from L. plantarum WCSF1 and a heterologous promoter derived from L. buchneri CD034 with a high and a low copy number origin of replication we demonstrated various expression levels of the model protein mCherry. All promoters were feasible for protein expression and in all cases, the high copy number origin of replication increased expression twofold. We found that the optimal spacer between the Shine-Dalgarno sequence and the start codon in L. plantarum consists of 8 nucleotides and elongation as well as shortening this sequence gradually down-regulates gene expression.

Conclusions: We have evaluated the effects of a set of gene regulatory tools to fine tune recombinant gene expression in L. plantarum CD033. We have thus, provided potential expression vectors useful for constitutive protein expression in lactic acid bacteria ranging from moderate to strong production levels.
\end{abstract}

Keywords: Lactobacillus plantarum CD033, Lactobacillus buchneri CD034, Constitutive promoter, Promoter strength, Elongation factor Tu, Ribosomal binding site, BioLector ${ }^{\mathrm{TM}}$

\section{Background}

Lactic acid bacteria (LAB) are responsible for various fermentation processes leading to food and feed preservation and improvement in flavour and texture of the fermented substrate [1]. Furthermore, many LAB have been found to be beneficial intestinal microbes associated with human and animal health [2]. Thus, LAB constitute an attractive tool for many applications in food and feed production [3-5], biotechnology [6-9] and medicine [10,11]. Besides using various wild-type $\mathrm{LAB}$, the possibility to expand the genetic repertoire of beneficial strains by genetic engineering becomes more and more attractive. Today, different gene expression systems are available for LAB, many of

\footnotetext{
* Correspondence: stefan.heinl@boku.ac.at

Christian Doppler Laboratory for Genetically Engineered Lactic Acid Bacteria, University of Natural Resources and Life Sciences, Vienna, Department of Biotechnology, Muthgasse 11, Vienna 1190, Austria
}

them optimized for Lactococcus lactis [12-15]. Inducible systems allow gene regulation by different additives such as lactose, xylose or other changing parameters like $\mathrm{pH}$ or temperature [16]. Another inducible expression system is based on the bacteriocin operon of Lactobacillus sakei which was shown to drive high-level gene expression in $L$. sakei and Lactobacillus plantarum [17]. Another bacteriocin induced system is the so called NICE-system (nisincontrolled gene expression system, for review see Mierau and Kleerezebem [18]), which was also adapted for use in L. plantarum [19]. Inducible expression systems are important when aiming at the overproduction of proteins to a maximum level, when proteins are toxic, or interfere in some other way with the host's metabolism. LAB comprise a food grade background that by genetic engineering may be equipped with additional enzymatic activities that would be beneficial during the process of food and feed 
fermentation [20], for the production of food additives $[6,7,21]$ or in the intestinal environment [22]. For these applications, inducible expression is not feasible; instead, constitutive promoters providing expression of a target gene at a suitable level are desirable. For example, the homologous lactate dehydrogenase promoter was recently used to constitutively express oxalate decarboxylase in L. plantarum WCFS1 [22]. It was shown in different studies, that although, bacterial promoters share similar features, promoter strength is strain and context specific and can vary significantly within LAB $[23,24]$. Therefore, it is necessary to identify and characterize promoters and regulatory sequences for each new host.

Besides promoter activity, also plasmid copy numbers have a major impact on recombinant protein expression. Most of the commonly used plasmid backbones are based on low copy number origins of replication (p256) [17] or high copy number origins of replication (pSH71) [25]. While in the case of $\beta$-glucuronidase expression, a high copy number plasmid lead to increased expression as compared to using a low copy number plasmid, for a second reporter protein, aminopeptidase $\mathrm{N}$, no such effect could be achieved [17]. This phenomenon was explained by gene specific properties and should be taken into consideration. Plasmid copy numbers were determined and estimated to be around three for p256 and 200 for pSH71. Another high copy number plasmid (pCD034-1) was isolated from a Lactobacillus buchneri strain, and its origin of replication was shown to support plasmid maintenance in L. plantarum [26]. The relative copy number of a pCD034-1derivative, pCDLbu-1, was estimated to exceed 200 copies per chromosome [27]. Besides transcription and gene dosage, also translation can be a major bottle neck and design of the ribosomal binding site strongly influences protein production levels $[28,29]$.

The goal of this study was to identify and evaluate simple tools and measures to fine-tune recombinant protein expression in $L$. plantarum with the purpose to provide suitable constitutive systems for applications in e.g. feed silage, food fermentation or in vivo drug delivery. Therefore, we compared different autologous and heterologous promoters, the impact of high and low copy number plasmid backbones and the influence of the distance between the Shine-Dalgarno sequence and the translation start signal. Our expression host was $L$. plantarum CD033. This strain has been isolated from a grass silage in Austria and may be used as an efficient starter culture for this purpose. In addition, L. plantarum CD033 was previously described to be feasible for highly efficient transformation with non-methylated DNA, allowing direct transfer of a ligation mix or assembled PCR fragments [30]. Therefore, intermediate hosts such as L. lactis or E. coli for high yield plasmid production are no longer required, which allows us very fast plasmid construction and manipulation, ideal for testing a large set of genetic elements.

\section{Results and discussion}

\section{Comparison of promoter activities: vector design}

Since no amplification of shuttle vectors in $E$. coli was required, all plasmids were designed without any additional $E$. coli specific origin of replication or selection markers. In the first experiment, we included four different constitutive promoters and tested for cytoplasmic expression of the reporter gene mCherry. The strong $\mathrm{P}_{11}$ promoter, a synthetic sequence based on an rRNA promoter from L. plantarum WCSF1 [31] was previously shown to be one of the strongest promoters, active in L. plantarum as its transcriptional activity was comparable to the inducible pSIP-based expression system [32]. Another beneficial feature of this promoter is its cross species activity, which has been shown for $L$. sakei [31]. Further, the promoter regions upstream of the gene encoding the putative translation elongation factor $\mathrm{TU}$ $\left(\mathrm{P}_{\text {tuf }}\right)$ from L. plantarum CD033 $\left(\mathrm{P}_{\text {tuf33 }}\right)$ and from L. buchneri CD034 $\left(\mathrm{P}_{\text {tuf34 }}\right.$, this study, see Figure 1$)$ were isolated and tested. Based on the fact that transcription elongation factors are among the most abundant proteins in bacteria, our assumption was that the corresponding promoters would induce strong transcription. We compared $\mathrm{P}_{\text {tuf-pro- }}$ moters from two different species in order to evaluate the versatility of this type of promoters and the feasibility for making shuttle vectors between $L$. plantarum and L. buchneri. In addition, using a heterologous $\mathrm{P}_{\text {tuf-promoter would }}$ minimize possible impact by species specific regulation mechanisms. The upstream sequences of the putative elongation factor $\mathrm{P}\left(\mathrm{P}_{\text {efp }}\right.$, this study, Figure 2$)$ was isolated from $L$. buchneri CD034 and based on previous observations was identified as quite active in the context of L. buchneri (data not shown).

\section{Characterization of selected promoter active fragments}

Pretesting of the promotor activities was accomplished by monitoring fluorescence signals of $L$. plantarum CD033 cells carrying the pCD256 $\triangle$ Ec-based constructs using a Tecan ${ }^{\mathrm{Ts}}$ reader. Cells were cultivated and measurements were performed for $23 \mathrm{~h}$ (Figure 3). While the promoter $\mathrm{P}_{\text {efp }}$ was very weak, good expression could be achieved with both $\mathrm{P}_{\text {tuf }}$ promoters and the $\mathrm{P}_{11}$ promoter. The $\mathrm{P}_{\text {efp }}$ promoter has previously been tested in L. buchneri CD034 and showed medium to high expression of mCherry (data not shown), indicating that its low activity is a species specific effect, and in the context of L. plantarum this promoter is not feasible for further experiments.

\section{Impact of plasmid copy number}

It has been shown for several plasmid based expression systems, that gene copy numbers have a strong influence on 


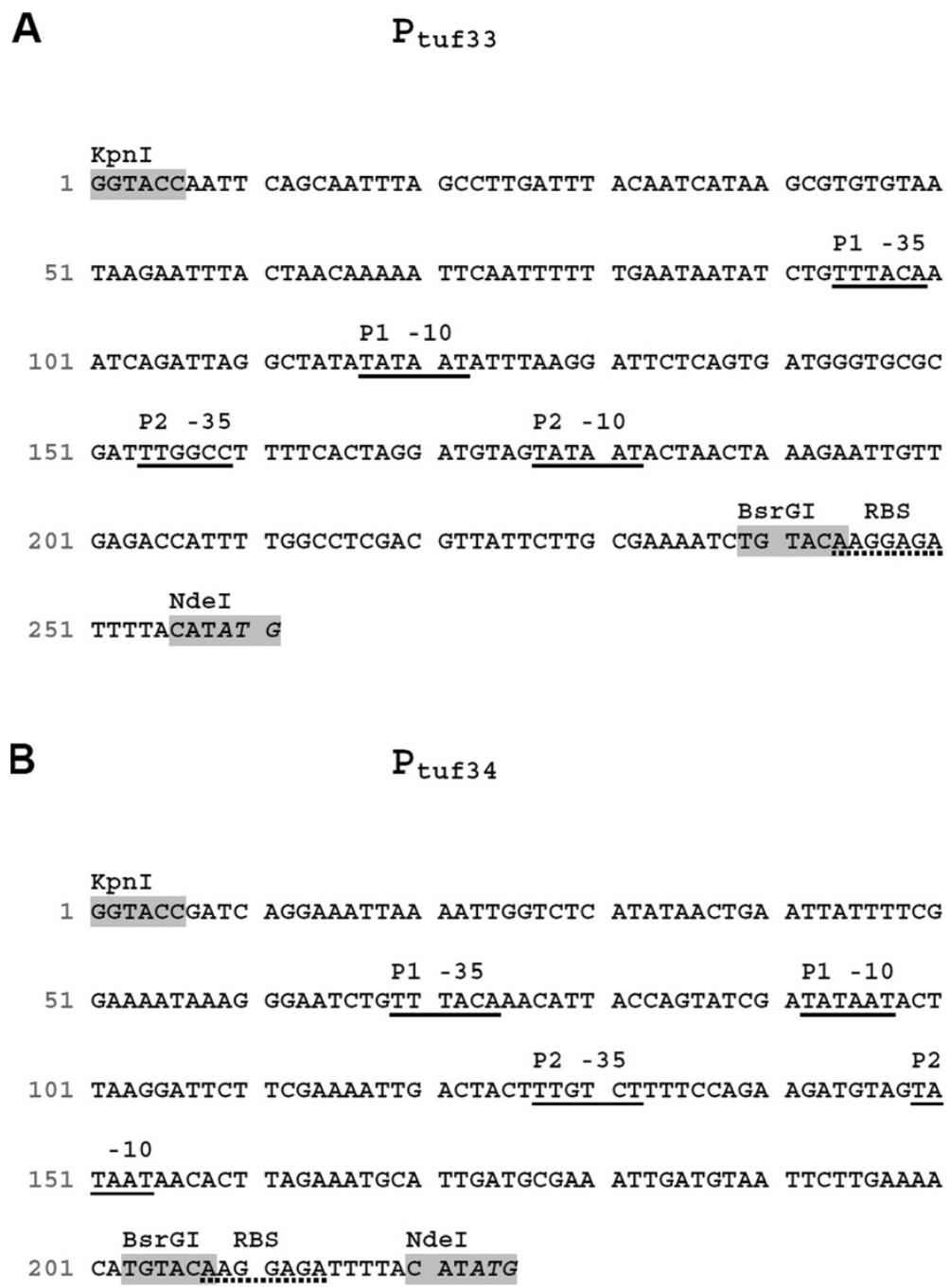

B

$P_{\text {tuf3 } 4}$

KpnI

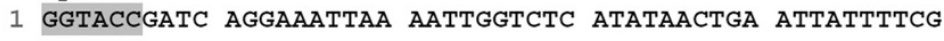

P1 -35

P1 -10

51 GAAAATAAG GGAATCTGTT TACAAACATT ACCAGTATCG ATATAATACT

P2 - 35

101 TAAGGATTCT TCGAAAATG ACTACTTTGT CTTTTCCAGA AGATGTAGTA

$-10$

151 TAATAACACT TAGAAATGCA TTGATGCGAA ATTGATGTAA TTCTTGAAAA

BsrGI RBS NdeI

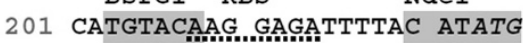

Figure 1 Nucleotide sequences of promoters $P_{\text {tuf33 }}(\mathbf{A})$ and $P_{\text {tuf34 }}(\mathbf{B})$. Both promoters are putative tandem promoters, each consisting of two consecutive promoter regions $\mathrm{P} 1$ and $\mathrm{P} 2$. The -35 and -10 regions are underlined, RBSs are underlined dotted, translation start signals are written in italics, restriction sites are highlighted in gray.

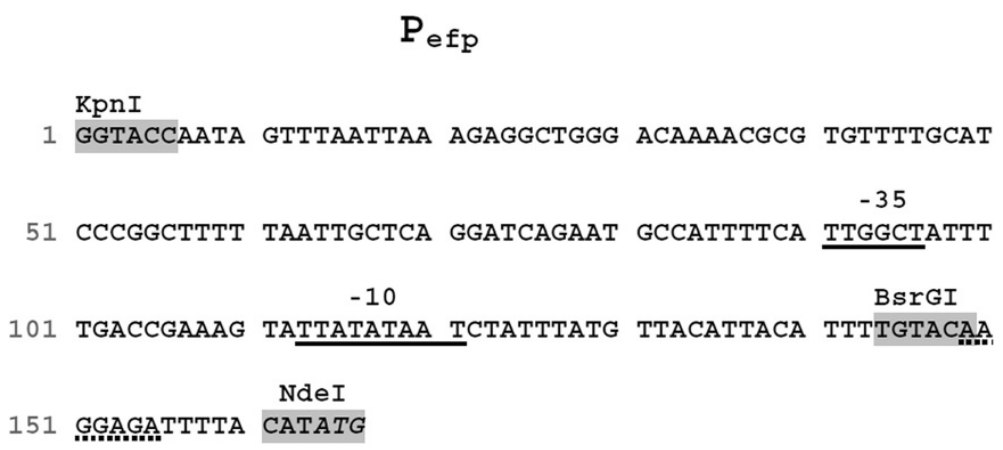

Figure 2 Nucleotide sequence of promoter $\mathbf{P}_{\text {efp. }}$. Ribosomal binding site, -35 and -10 regions are underlined, translation start signal is written in italics, restriction sites are highlighted in gray. 


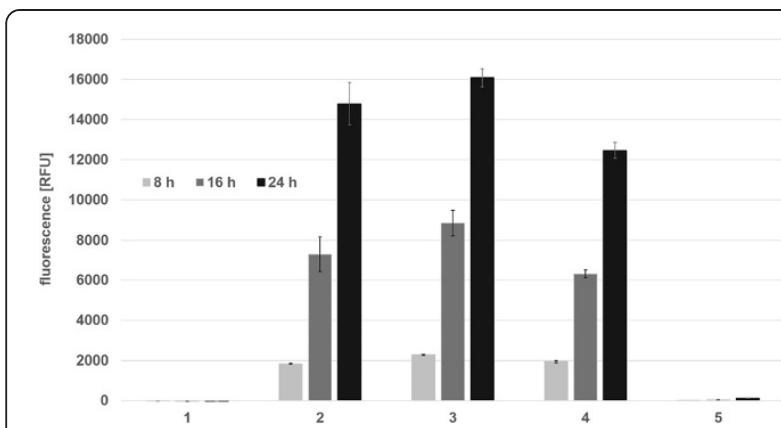

Figure 3 Tecan $^{\text {rm }}$ reader measurement of mCherry expression levels in L. plantarum CD033 carrying the pCD256 $\mathrm{E}$ __mCherry vectors after 8, 16 and 23 hours. Error bars show standard deviation. (1) L. plantarum CD033 negative control pCD256 EC_mCherry, (2) L. plantarum CD033 pCD256 EEc_Ptuf33_mCherry, (3) L. plantarum CD033 pCD256 EC_Ptuf34_mCherry, (4) L. plantarum CD033

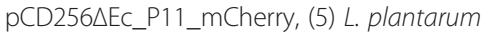
CD033 pCD256 $\triangle$ EC_Pefp_mCherry.

overall expression of a heterologous protein. While normally an increase leads to higher expression rates, sometimes too high replication rates can be detrimental to cell growth [33]. Most of the theta- or rolling circle replicating plasmids normally used in L. plantarum strains have copy numbers between one and five. Yet for the pSIP411-based expression system also the high copy number origin of replication derived from $\mathrm{pSH71}$ is used [17]. In order to investigate the influence of plasmid copy number on the expression level, we constructed several plasmids, either containing the theta replicating, low copy number miniori p256 resulting in pCD256 $\triangle \mathrm{Ec}$ plasmid constructs, or the high copy number ori pCD034-1, isolated from L. buchneri CD034 [26] resulting in the plasmid pCDLbu- $1 \Delta \mathrm{Ec}$. Table 1 lists all constructs used for fermentation experiments using the BioLector" platform.

When looking at the growth rates of the tested clones (Figure 4), it becomes apparent that pCDLbu- $1 \Delta \mathrm{Ec}$ ${ }_{-} \mathrm{P}_{\text {tuf33_mCherry }}$ and pCDLbu-1 $1 \Delta \mathrm{Ec}_{-} \mathrm{P}_{\text {tuf34_mCherry, }}$

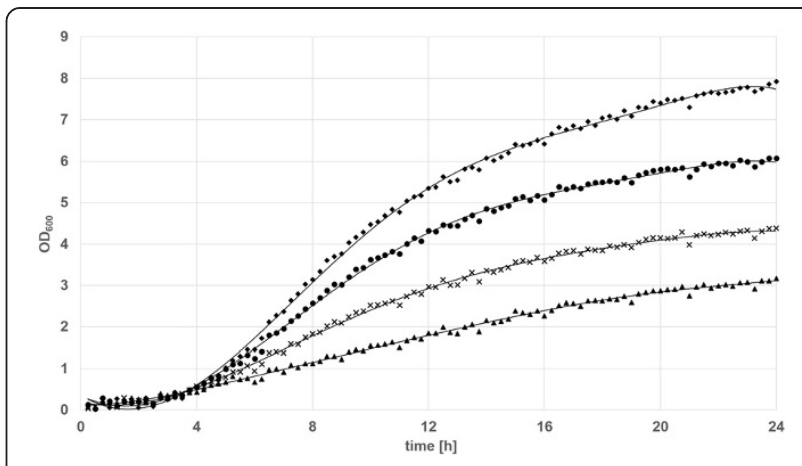

Figure 4 Growth rates of $L$. plantarum $\mathrm{CD} 033$ carrying the pCDLbu-1 $1 \Delta$ Ec_mCherry vectors determined with the BioLector ${ }^{\mathrm{rm}}$. (•) L. plantarum CD033 negative control pCDLbu-1 $\triangle E$ E_mCherry, $(\bullet) L$. plantarum CD033 pCDLbu-1 $\Delta$ Ec_P11_mCherry, (x) L. plantarum CD033

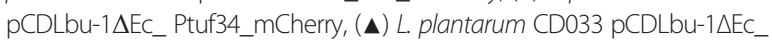
Ptuf33_mCherry.

both containing the high copy number origin of replication, produce less biomass during fermentation. This might be because, due to overproduction of mCherry, the overall metabolic load hampers the growth rate. Alternatively, the high number of $\mathrm{P}_{\text {tuf-promoter copies }}$ may capture essential sigma factors, and the cell is unable to proceed with translation of homologous genes at a normal rate. When comparing the overall transcriptional activities (Figure 5), the $P_{11}$ promoter in combination with the high copy number plasmid backbone turned out to be the strongest, followed by the two other pCDLbu$1 \Delta \mathrm{Ec}$-based constructs. All fermentations based on the theta replicating plasmids produced about half the fluorescence signal as compared to their rolling circle replicating counterpart (Figure 6). Growth rates were comparable for all pCD256 $\triangle$ Ec-based constructs (data not shown). Specific gene expression as shown in Figure 7A reflects the impact of growth inhibition in case of the high copy number constructs, while for the low copy number plasmids, specific expression rates were comparable (Figure 7B).

Table 1 Description of constructs indicating the promoter and origin of replication present on each plasmid

\begin{tabular}{|c|c|c|c|c|}
\hline Construct & Promoter & Promoter from & Reference & Origin of replication from \\
\hline pCDLbu-1 $\triangle$ Ec_mCherry (neg. contr.) & None & w/o promoter & This study & pCD034-1 \\
\hline pCD256 EEc_mCherry (neg. contr.) & None & w/o promoter & This study & p256 \\
\hline pCDLbu-1 $\triangle$ Ec_Pefp_mCherry & $P_{\text {efp }}$ & L. buchneri CD034 & This study & pCD034-1 \\
\hline pCD256 $\triangle E$ E_Pefp_mCherry & $P_{\text {efp }}$ & L. buchneri CD034 & This study & p256 \\
\hline pCDLbu-1 $\triangle$ Ec_Ptuf33_mCherry & $P_{\text {tuf33 }}$ & L. plantarum CD033 & This study & pCD034-1 \\
\hline pCD256 $\Delta$ Ec_Ptuf33_mCherry & $P_{\text {tuf33 }}$ & L. plantarum CD033 & This study & p256 \\
\hline pCDLbu-1 $\triangle$ Ec_Ptuf34_mCherry & $P_{\text {tuf34 }}$ & L. buchneri CD034 & This study & pCD034-1 \\
\hline pCD256 EEc_Ptuf34_mCherry & $P_{\text {tuf34 }}$ & L. buchneri CD034 & This study & p256 \\
\hline pCDLbu-1 $\triangle$ Ec_P11_mCherry & $P_{11}$ & L. plantarum library & {$[31]$} & pCD034-1 \\
\hline 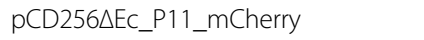 & $P_{11}$ & L. plantarum library & {$[31]$} & p256 \\
\hline
\end{tabular}




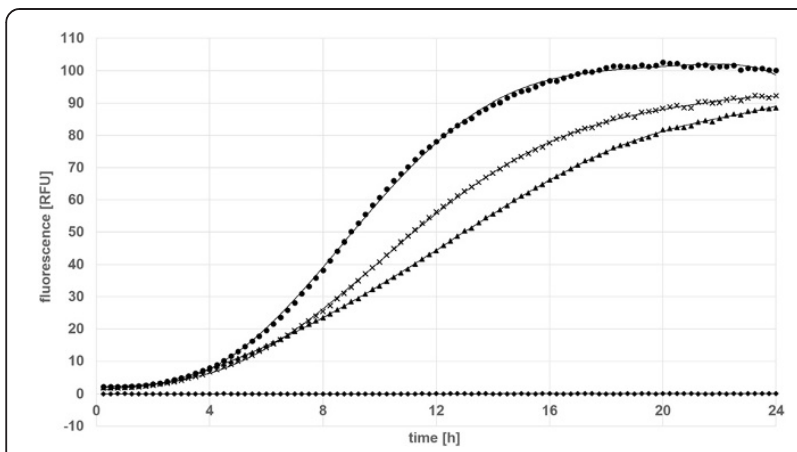

Figure 5 BioLector $^{\mathrm{tm}}$ analysis of mCherry expression levels in $L$. plantarum $\mathrm{CD} 033$ carrying the $\mathrm{pCDLbu}-1 \Delta \mathrm{Ec}$ _mCherry constructs. $(\bullet)$ L. plantarum CD033 pCDLbu-1 $\triangle E C$ EP11_mCherry,

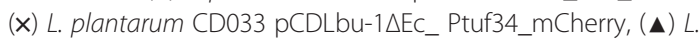
plantarum CD033 pCDLbu-1 $\triangle E$ E__Ptuf33_mCherry, ( $\downarrow$ ) L. plantarum CD033 negative control pCDLbu-1 $1 \mathrm{EE}$ _mCherry.

\section{Influence of the relative position of the Shine-Dalgarno sequence}

The specific base pairing between the 3 '-end of the rRNA and the sequence preceding an initiator AUG provides a mechanism by which the cell can distinguish between initiator AUGs and internal and/or out-of-phase AUG sequences. The degree of base pairing also plays a role in determining the rate of initiation at different AUG initiator codons in polycistronic mRNAs [34]. The ribosomal binding site (RBS) or Shine-Dalgarno-sequence (SDS) used in our expression constructs was originally derived from pSIP409. This sequence, AAGGAGA [31], however, did not correspond to the perfect matching SDS core sequence AAGGAGG, identified in Lactobacillus plantarum. Therefore, the RBS of the $\operatorname{slpB}$ gene from $L$. buchneri CD034, which fits better to the SDS core sequence and corresponds to the most abundantly expressed gene in $L$. buchneri CD034 was chosen for RBS-optimization (Table 2, SDOPT\#9). The distance between the SDS and the start codon of our constructs comprised 9 nucleotides. For fine-tuning translational efficiency we changed the SDS to the perfect match sequence and varied the distance between the SDS and the translational start-site, analysing the range between 5 and 12 nucleotides (Table 2). All constructs were based on the low copy p256 origin of replication and mCherry expression was under control of the $\mathrm{P}_{11}$ promoter. The low copy origin of replication was chosen for these experiments in order to provide expression levels that can be up-regulated without causing growth hampering stress due to over-production.

Growth rates were comparable for all constructs (data not shown). Figure 8 shows the correlation between differences in fluorescent signals and the varied length of the spacer sequences. The highest expression was detected after $18 \mathrm{~h}$ of cultivation. Spacer sequences shorter than 7 nucleotides turned out to considerably hamper

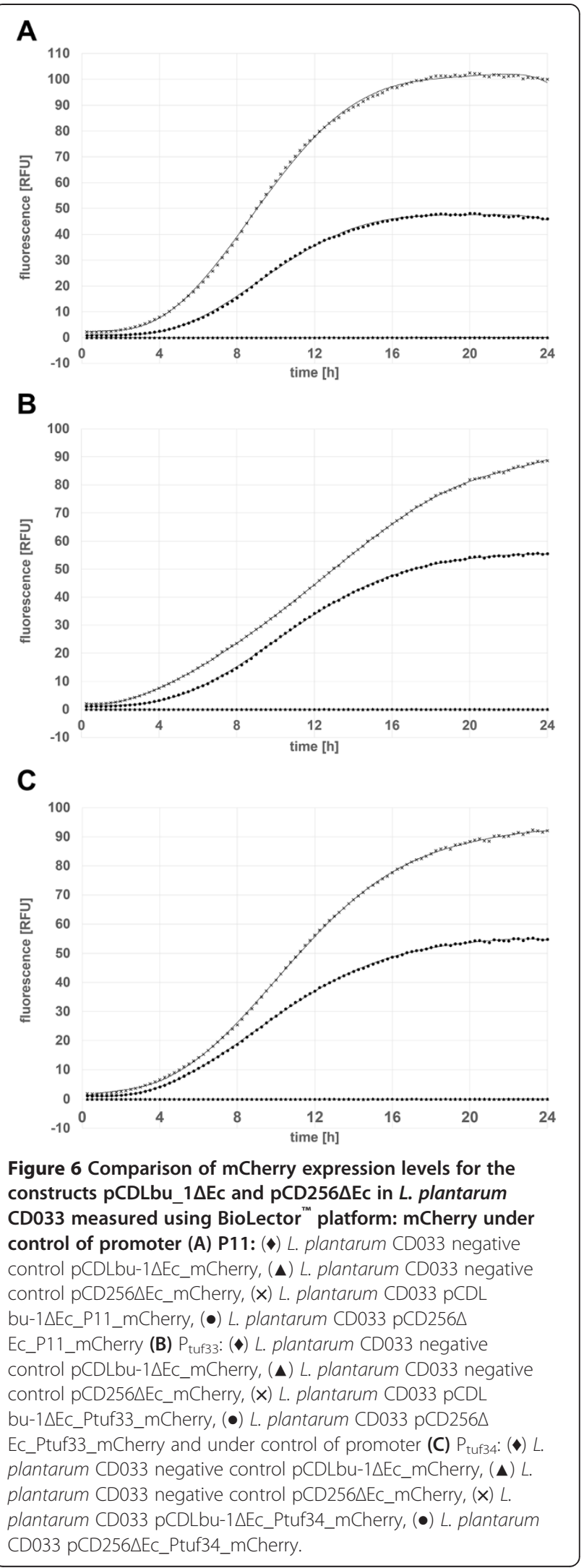



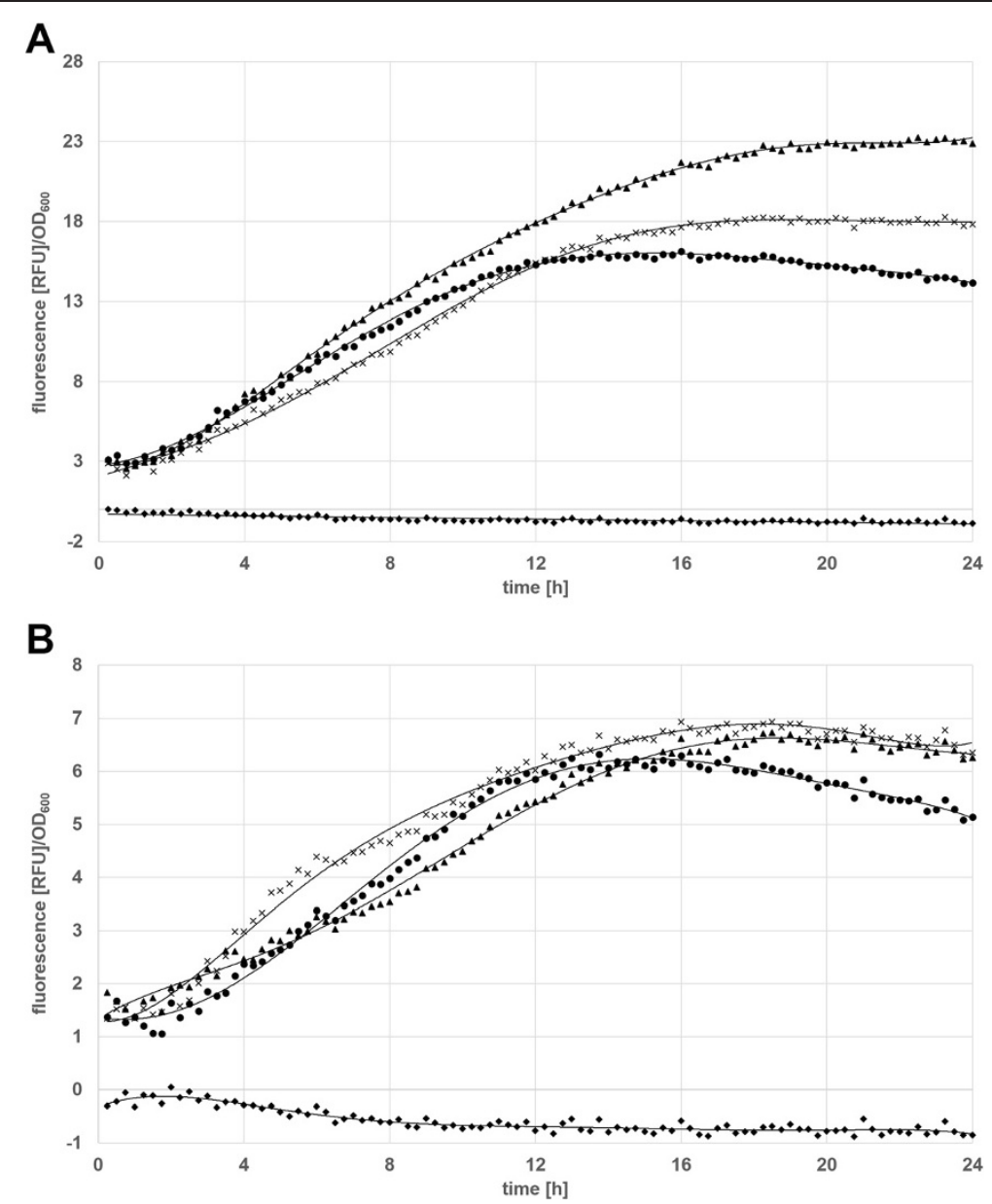

Figure 7 Specific expression rates of the pCDLbu_1 $1 \Delta \mathrm{Ec}$ and pCD256 $\triangle \mathrm{Ec}$ constructs in L. plantarum CD033 determined with the

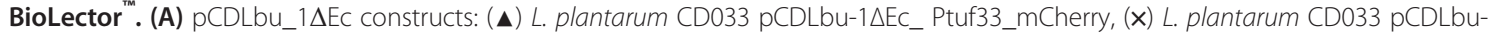

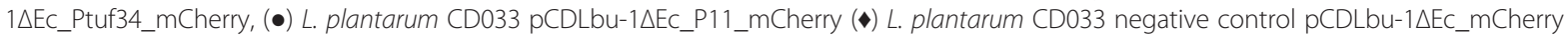

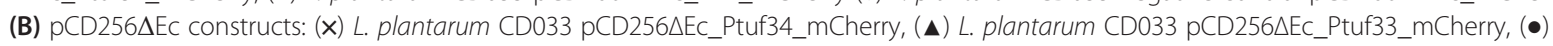

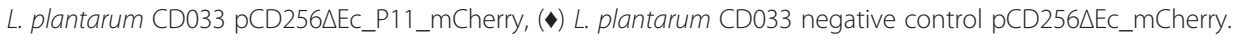

Table 2 List of constructs with varying spacer sequences between the SDS and the start codon

\begin{tabular}{ll}
\hline Construct & SDS-spacer-start codon \\
\hline SDOPT\#5 & AAGGAGG AATAC ATG \\
SDOPT\#6 & AAGGAGG AAATAC ATG \\
SDOPT\#7 & AAGGAGG AAATTAC ATG \\
SDOPT\#8 & AAGGAGG AAATTTAC ATG \\
SDOPT\#9 & AAGGAGG AAATTATAC ATG \\
SDOPT\#10 & AAGGAGG AAAATTATAC ATG \\
SDOPT\#11 & AAGGAGG AAAAATTATAC ATG \\
SDOPT\#12 & AAGGAGG AAAAAATTATAC ATG \\
\hline
\end{tabular}

Spacer sequences are written in bold. translation efficiency, while 8 nucleotides seemed to be optimal. A slight decrease could be observed when the spacer was designed to be as long as 12 nucleotides. Thus, if desired, recombinant expression may be downregulated by using spacer variations shorter than 7 or longer than 11 nucleotides. Fine-tuning of protein expression in order to utilize a host cell in an optimal way can be realized by regulation of several parameters. Here, we investigated and demonstrated the impact of transcriptional activity, gene copy number and translation efficiency for the species Lactobacillus plantarum. Considering that many strains of this species are used as highly beneficial starter cultures for food and feed applications, the potential applications are manifold. Overexpression of cellulases and hemicellulases could contribute to digestibility and quality of grass silage. Moreover, new substrates, such as leaves or other plant waste material could be fermented more efficiently and fed into biogas plants. 


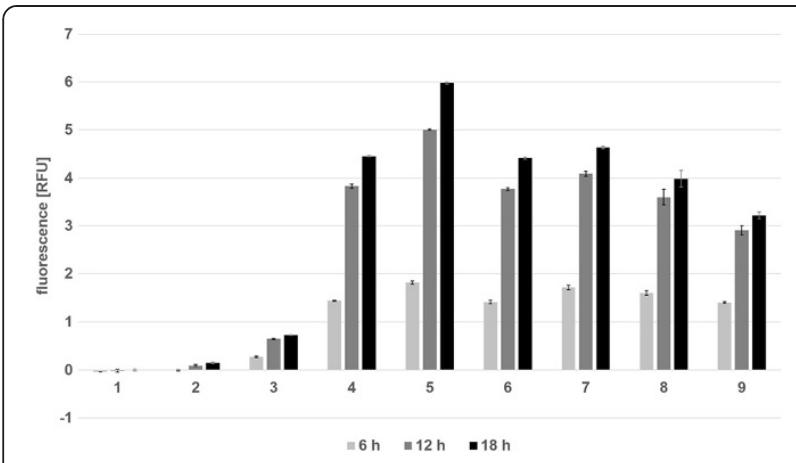

Figure 8 BioLector $^{\mathrm{Tm}}$ analysis of the SDS-varied pCD256 $\mathrm{EE}$ c mCherry constructs after 6,12 and 18 hours. Error bars show standard deviation. (1) L. plantarum CD033 negative control pCD256 2 Ec_mCherry, (2) L. plantarum CD033 pCD256 C_P11_mCherry SDOPT\#5, (3) L. plantarum CD033 PCD256 C_P11_mCherry SDOPT\#6, (4) L. plantarum CD033 pCD256 $\triangle$ Ec_P11_mCherry SDOPT\#7, (5) L. plantarum CD033 pCD256 $\triangle$ Ec_P11_mCherry SDOPT\#8, (6) L. plantarum CD033 pCD25

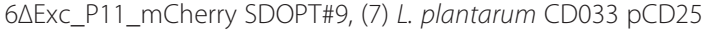

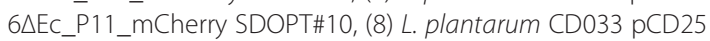

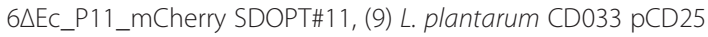
$6 \triangle E C \_P 11 \_m C h e r r y ~ S D O P T \# 12$

Another application is the food-grade expression of enzymes for making nutritional additives, e.g. ß-galactosidase or chitinase are used to produce oligo-saccharides [6,7]. Finally, $L$. plantarum is a widely spread probiotic and as such may be used as a scaffold for vaccination or treatment of intestinal diseases in vivo. Next steps are to test different strains within the species of $L$. plantarum as well as different genes with biotechnological potential.

\section{Conclusions}

Lactobacillus plantarum is widely spread in nature. It is used as a highly effective silage additive, has probiotic properties and serves as a cell factory to produce recombinant proteins. Here we have tested several constitutive promoters in combination with high and low copy number plasmid backbones in L. plantarum CD033. Thereby, we confirmed the previously described promoter $\mathrm{P}_{11}$ [31] to be feasible for strong constitutive protein expression, especially in combination with a high copy number origin of replication. We further characterized two new promoters, $\mathrm{P}_{\text {tuf33 }}$ and $\mathrm{P}_{\text {tuf34, }}$, which now are available as additional candidates to drive constitutive expression in L. plantarum as well as in L. buchneri. The impact of different origins of replication was investigated, demonstrating twofold higher product yields for the pCDLbu$1 \Delta$ Ec-based constructs containing the high copy number origin of replication derived from the L. buchneri CD034 plasmid pCD034-1 [26]. Besides controlling transcriptional levels and gene copy number, we evaluated the possibility to up or down-regulate the overall target gene expression by varying the distance between the SDS and the start-codon. We could show that in L. plantarum CD033 there was a direct correlation between these two parameters, reaching the highest expression levels when the spacer spanned 8 nucleotides. While the performance and behavior of expression regulatory elements might differ in dependence of the target gene, predictions about their impact will facilitate vector design strategies and experimental set-ups in the future. Overall, we believe that the silage strain L. plantarum CD033 as well as the L. plantarum species in general is a highly versatile tool for improving nutrition quality, human health and biomass based energy production.

\section{Methods}

\section{Cultivation and transformation of L. plantarum CD033}

The Lactobacillus plantarum strain CD033 was grown in de Man-Rogosa-Sharpe (MRS) medium [35] at $30^{\circ} \mathrm{C}$ under oxygen limitation, supplemented with chloramphenicol $\left(10 \mu \mathrm{g} \mathrm{ml}^{-1}\right)$ if required. The transformation of plasmids into L. plantarum CD033 was accomplished according to the electroporation protocol described earlier [27].

\section{DNA techniques and cloning procedure}

All Enzymes were purchased from New England Biolabs (NEB, USA). DNA fragments were amplified using the Phusion High-Fidelity DNA Polymerase according to the manufacturer's recommendations. All resulting clones were colony screened using OneTaq DNA Polymerase as recommended by the producer. All PCRs were carried out with a C1000 Thermal Cycler (BioRad, USA). Restriction digests were performed following the manufacturer's instructions. PCR products were purified using the NucleoSpin Gel and PCR Clean-up Kit (MachereyNagel, Germany). Ligations were performed using T4ligase. All primers are listed in Table 3.

\section{Construction of expression vectors for promoter activity testing}

A gene, codon optimized for L. plantarum WCFS1 using the webtool JCat (http://www.jcat.de/), encoding the red fluorescent protein mCherry was synthesized as a gBlock (IDT, Belgium) and amplified using the primers mCherry_F (NdeI)/mCherry_R (BamHI). Promotor $\mathrm{P}_{11}$ was also amplified from a gBlock using the primers P11_F (SacI, KpnI)/P11_R (NdeI). The two PCR products were digested with NdeI and ligated one with each other to gain the DNA-fragment P11_mCherry. The ligation product was again amplified using the primers P11_F (SacI,KpnI)/mCherry_R (BamHI).

\section{Theta-replicating pCD256 $\Delta \mathrm{Ec}$-constructs}

For construction of the theta-replicating expression vectors the plasmid pCD256 $\triangle \mathrm{EC}$ _hTTF1 [30] was amplified using the primers sCAT_R (KpnI)/Tldh_F (BamHI). The PCR 
Table 3 List of primers used in this study

\begin{tabular}{|c|c|}
\hline Primer & Sequence $5^{\prime}->3^{\prime}$ \\
\hline mCherry_F (Ndel) & CAGCAGCAGCATATGTTATCAAAGGGTGAAGAAG \\
\hline mCherry_R (BamHI) & CGTCGTGGATCCTTATCACTTGTATAATTCATCCATACC \\
\hline P11_F (Sacl,Kpnl) & GACGACGAGCTCGGTACCTTACAGCTCCAGATCTAGCG \\
\hline P11_R (Ndel) & GACGACGACCATATGTAAAATCTCCTTGTAATAGTATTT \\
\hline sCAT_R (Kpnl) & GCTGCTGGTACCGGGCAGGTTAGTGACATTAG \\
\hline Tldh_F (BamHI) & CTGCTGGGATCCAAAACCGCTGT \\
\hline mCherry_F (BsrGl) & CAGCAGTGTACAAGGAGATTTAACATATGTTATCAAAGGGTGAAG \\
\hline Ptuf_CD033_F (Kpnl) & GACGACGGTACCAATTCAGCAATTTAGCCTTGATTTAC \\
\hline Ptuf33_R (BsrGl) & GTCCAGTGTACAGATTTTCGCAAGAATAACGTCG \\
\hline Ptuf_CD034_F (Kpnl) & GTCGTCGGTACCGATCAGGAAATTAAAATTGGTCTC \\
\hline Ptuf34_R (BsrGl) & GTCGTCTGTACATGTITTCAAGAATTACATCAATTTCG \\
\hline efp-sense_F (Mfel, Kpnl) & GCAGCACAATTGGGTACCAATAGTTTAATTAAAGAGGCTGG \\
\hline Pefp_R (BsrGl) & CAGCAGTGTACAAAATGTAATGTAACA \\
\hline Cat_F (Nhel) & CGACGAGCTAGCAATGTGGTCTITATTCTTCAAC \\
\hline M13_R (Nhel) & CGACGAGCTAGCAGCCAGGAAACAGCTATGACC \\
\hline Tldh_amp_R (Pstl) & CTGCTGCTGCAGAAAAAGATTAAAAAAGCCGCTGC \\
\hline P11_control_R (Kpnl) & CTGCACGGTACCCAAGGAGATTTTACATATGTTATCA \\
\hline Cat_seq2_back & TACATCATTCTGTTTGTGATGG \\
\hline 4_6_n2_R & AACTCATAATACGCCTAAGCC \\
\hline EFP_screen_back & GATTCCCGATAACAACCGT \\
\hline SDOPT_5_F (Xbal) & ACGACGTCTAGATAAGGAGGAATACATGTTATCAAAGGGTGAAGAAG \\
\hline SDOPT_6_F (Xbal) & ACGACGTCTAGATAAGGAGGAAATACATGTTATCAAAGGGTGAAGAAG \\
\hline SDOPT_7_F (Xbal) & ACGACGTCTAGATAAGGAGGAAATTACATGTTATCAAAGGGTGAAGAAG \\
\hline SDOPT_8_F (Xbal) & ACGACGTCTAGATAAGGAGGAAATTTACATGTTATCAAAGGGTGAAGAAG \\
\hline SDOPT_9_F (Xbal) & ACGACGTCTAGATAAGGAGGAAATTATACATGTTATCAAAGGGTGAAGAAG \\
\hline SDOPT_10_F (Xbal) & ACGACGTCTAGATAAGGAGGAAAATTATACATGTTATCAAAGGGTGAAGAAG \\
\hline SDOPT_11_F (Xbal) & ACGACGTCTAGATAAGGAGGAAAAATTATACATGTTATCAAAGGGTGAAGAAG \\
\hline SDOPT_12_F (Xbal) & ACGACGTCTAGATAAGGAGGAAAAAATTATACATGTTATCAAAGGGTGAAGAAG \\
\hline SDOPT_R (Xbal) & ACGACGTCTAGAGAATACATATATGCTGGCCAGC \\
\hline
\end{tabular}

Restriction sites are written in bold letters.

product was double digested with restriction endonucleases KpnI and BamHI and fragment P11_mcherry was ligated into the vector backbone. The resulting construct was designated pCD256 $\triangle \mathrm{EC}$ _P11_mCherry.

To get constructs with the other promoters, $\mathrm{pCD} 256 \triangle \mathrm{EC}$ _P11_mcherry was amplified using the primers mCherry_F (BsrGI)/sCAT_R (KpnI). The promoters were amplified using the primers Ptuf_CD033_F (KpnI)/Ptuf33_R (BsrGI) for promoter $\mathrm{P}_{\text {tuf33 }}$, Ptuf_CD034_F (KpnI)/Ptuf34_R (BsrGI) for promoter $\mathrm{P}_{\text {tuf34 }}$ and efp-sense_F (MfeI, KpnI)/Pefp_R (BsrGI) for the efp-promoter $\mathrm{P}_{\text {efp }}$. The $\mathrm{PCR}$ products were KpnI/BsrGI digested and each promotor was ligated with the vector backbone described above. The resulting constructs were pCD256 $\triangle \mathrm{Ec}_{-}$ Ptuf33_mCherry, pCD256 $\Delta$ Ec_Ptuf34_mCherry and
pCD256 $\Delta$ Ec_Pefp_mCherry. All constructs were introduced into $L$. plantarum CD033 by electroporation. The pCD256 $\triangle \mathrm{Ec}$ constructs were colony screened using the primers Cat_seq2_back and efp_screen_back. All constructs were confirmed by sequencing using the same primers (Microsynth, Switzerland).

\section{Rolling circle replicating (RCR) pCDLbu- $1 \Delta E c$-constructs}

For the RCR-constructs plasmid pCDLbu-1 [26] served as vector backbone. First all E.coli-specific sequences were removed by PCR using the primers Cat_F (NheI)/ M13_R (NheI). After NheI digestion the amplicon was recircularized by selfligation and transformed directly into $L$. plantarum CD033. The resulting vector was designated pCDLbu-1 $\triangle \mathrm{EC}$ and was subsequently amplified 

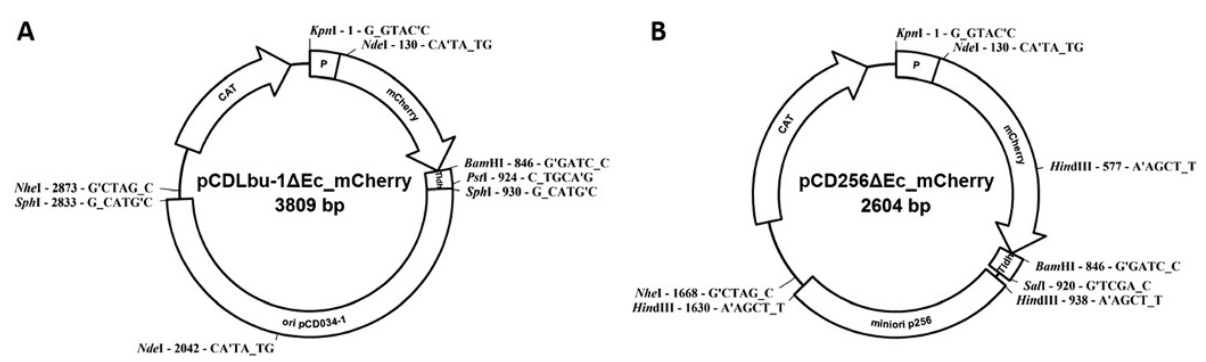

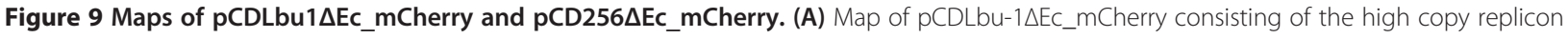
from plasmid pCD034-1 (Heinl et al. 2011), a chloramphenicol resistance gene for selection in LAB, the mCherry expression cassette including one of the chosen promotors, an mCherry reporter gene and the T/dh terminator from L. casei (Spath et al. 2012b). (B) Map of pCD256 EC__mCherry containing the minimal origin of replication from plasmid p256, the chloramphenicol resistance gene, and the mCherry expression cassette.

using the primers sCAT_R (KpnI)/Tldh_F (BamHI) and digested with KpnI/PstI. The already finished pCD256 $\mathrm{Ec}$ vectors served as template for insert amplification. Therefore, forward primers P11_F (SacI/KpnI), efp-sense_F (MfeI,KpnI), Ptuf_CD033_F (KpnI), Ptuf_CD034_F (KpnI) and the reverse primer Tldh_amp_R (PstI) were used to obtain the expression cassettes P11_mCherry_Tldh, Pefp_mCherry_Tldh, Ptuf33_mCherry_Tldh and Ptuf34_mCherry_Tldh. After a KpnI/PstI digest the inserts were ligated with the pCDLbu-1 $\triangle \mathrm{Ec}$ backbone to gain the constructs pCDLbu-1 $\Delta \mathrm{Ec}$ _P11_mCherry, pCDLbu-1 $\Delta \mathrm{Ec}$ Pefp_mCherry, pCDLbu-1 $\Delta$ Ec_Ptuf33_mCherry and pCDL bu-1 $\Delta$ Ec_Ptuf34_mCherry which were used to transform $L$. plantarum CD033 by electroporation. The pCDLbu1 $\Delta \mathrm{Ec}$ constructs were colony screened using the primer pair Cat_seq2_back /4_6_n2_R. All constructs were confirmed by sequencing using the same primers (Microsynth, Switzerland).

The general vector designs are shown in Figure 9.

\section{Cloning of negative controls}

Plasmids pCDLbu1 $\Delta$ Ec_mCherry and pCD256 $\Delta$ Ec_mCherry lacking a promoter upstream of the mCherry gene served as negative controls. Therefore, plasmids pCDLbu1$\triangle \mathrm{Ec} \_\mathrm{P} 11 \_\mathrm{mCherry}$ and the pCD256 $\Delta \mathrm{Ec}$ _P11_mCherry were amplified using the primers P11_control_R (KpnI)/ sCAT_R (KpnI). After a KpnI-digest the PCR products were self-ligated and used to transform L. plantarum CD033.

\section{Constructs for Shine-Dalgarno Optimization}

Plasmid pCD256_P11_mCherry was used as PCR template for this experiment. Constructs were amplified using the forward primers SDOPT_5_F (XbaI), SDOPT_6_F (XbaI), SDOPT_7_F (XbaI), SDOPT_8_F (XbaI) SDOPT_9_F (XbaI), SDOPT_10_F (XbaI), SDOPT_11_F (XbaI) and SDOPT_12_F (XbaI) and the reverse primer SDOPT_R (XbaI). Restriction digests with $\mathrm{XbaI}$ were performed. The DNA-fragments were self-ligated and used to transform $L$. plantarum CD033.
Colonies resistant to chloramphenicol were screened by PCR using the primers Cat_seq2_back/EFP_screen_back and correctness of the constructs was confirmed by sequencing of the obtained PCR-products (Microsynth, Switzerland).

\section{Determination of mCherry expression by $\operatorname{Tecan}^{\mathrm{TM}}$ reader measurement}

The Infinite M1000 Tecan $^{\mathrm{TM}}$ reader connected to the Tecan i-control 1.6 software (Tecan Group Ltd., Switzerland) was used for pretesting. Overnight cultures were diluted to an $\mathrm{OD}_{600}$ value of $0.1 .200 \mu \mathrm{L}$ of each sample was pipetted into a 96 well clear bottom plate (Perkin Elmer, USA). The mCherry fluorescence at $587 \mathrm{~nm}$ was measured at $30^{\circ} \mathrm{C}$ every 30 minutes over $23 \mathrm{~h}$. A gain of 140 was used for fluorescence measurments. Immediately prior to fluorescence measurment, samples were shaken for 15 seconds. Samples were analyzed in quadruplicate.

\section{Determination of $\mathrm{mCherry}$ expression by BioLector ${ }^{\mathrm{Tm}}$ measurement}

mCherry measurements were accomplished using the BioLector $^{\mathrm{Tm}}$ Basic device (m2p-labs Germany). Data were analyzed using the BioLection 2.3.13 software (m2p-labs, Germany). Overnight cultures were diluted to an $\mathrm{OD}_{600}$ value of 0.1 and subsequently $800 \mu \mathrm{l}$ of each sample were pipetted into a MTP-48 FlowerPlate (m2p-labs, Germany). Fluorescence was determined using the EOP-119 LED module for mCherry at $580 \mathrm{~nm}$ and a gain of 80. Measurement was executed every 15 minutes, cells were cultivated at $30^{\circ} \mathrm{C}$ for 24 hours under constant shaking at 1,000 rpm. Negative controls were analyzed in duplicate, samples were analyzed in triplicate. For biomass analysis a calibration curve was generated. The $\mathrm{OD}_{600}$ values of a L. plantarum CD033 o/n cultures were measured undiluted, 1:1.3, 1:2, 1:3, 1:4, 1:5, 1:7, 1:10 and 1:20 diluted in an Implen Nano Photometer (Implen, Germany) and correlated with the scattered light data at $620 \mathrm{~nm}$ and a gain of 20 measured using the BioLector system. The linear equation of the 
standard curve was $\mathrm{y}=0.0312 \mathrm{x}-0.6465$ with a correlation coefficient $\mathrm{R}=0.9991$.

\section{Abbreviations}

$\mathrm{H}$ : Hours; O/N: Over Night; $\mathrm{OD}_{600}$ : Optical density at $600 \mathrm{~nm}$; RCR: Rolling circle replicating; RFU: Relative fluorescence units; RPM: Rounds per minutes; SDS: Shine-Dalgarno sequence; W/O: Without.

\section{Competing interests}

The authors declare that they have no competing interests.

\section{Authors' contributions}

The work presented here was carried out in collaboration between all authors. S Heinl and RG defined the research theme and designed the experiments. CT, EE and S Heiss carried out the laboratory experiments, analyzed the data, interpreted the results and prepared this manuscript with input, feedback and advice from S Heinl and RG. All authors have contributed to, seen and approved the manuscript.

\section{Acknowledgements}

This work was supported by the Christian Doppler Research Association Vienna, Austria.

Received: 21 August 2014 Accepted: 18 October 2014

Published online: 20 November 2014

\section{References}

1. Smid EJ, Kleerebezem M: Production of aroma compounds in lactic fermentations. Annu Rev Food Sci Technol 2014, 5:313-326.

2. labal MZ, Qadir MI, Hussain T, Janbaz KH, Khan YH, Ahmad B: Review: probiotics and their beneficial effects against various diseases. Pak $J$ Pharm Sci 2014, 27:405-415.

3. Eikmeyer FG, Kofinger P, Poschenel A, Junemann S, Zakrzewski M, Heinl S, Mayrhuber E, Grabherr R, Puhler A, Schwab H, Schluter A: Metagenome analyses reveal the influence of the inoculant Lactobacillus buchneri CD034 on the microbial community involved in grass ensiling. J Biotechnol 2013, 167:334-343.

4. Dogi CA, Fochesato A, Armando R, Pribull B, de Souza MM, da Silva Coelho I, Araujo de Melo D, Dalcero A, Cavaglieri L: Selection of lactic acid bacteria to promote an efficient silage fermentation capable of inhibiting the activity of Aspergillus parasiticus and Fusarium gramineraum and mycotoxin production. J Appl Microbiol 2013, 114:1650-1660.

5. Waters DM, Mauch A, Coffey A, Arendt EK, Zannini E: Lactic acid bacteria as a cell factory for the delivery of functional biomolecules and ingredients in cereal based beverages: a review. Crit Rev Food Sci Nutr 2013, 55:503-520.

6. Nguyen TT, Nguyen HA, Arreola SL, Mlynek G, Djinovic-Carugo K, Mathiesen $G$, Nguyen $T H$, Haltrich D: Homodimeric beta-galactosidase from Lactobacillus delbrueckii subsp. bulgaricus DSM 20081: expression in Lactobacillus plantarum and biochemical characterization. J Agric Food Chem 2012, 60:1713-1721.

7. Nguyen HA, Nguyen TH, Nguyen TT, Peterbauer CK, Mathiesen G, Haltrich D: Chitinase from Bacillus licheniformis DSM13: expression in Lactobacillus plantarum WCFS1 and biochemical characterisation. Protein Expr Purif 2012, 81:166-174.

8. Staudigl P, Haltrich D, Peterbauer CK: L-Arabinose isomerase and D-xylose isomerase from Lactobacillus reuteri: characterization, coexpression in the food grade host Lactobacillus plantarum, and application in the conversion of D-galactose and D-glucose. J Agric Food Chem 2014 62:1617-1624

9. Bohmer N, Lutz-Wahl S, Fischer L: Recombinant production of hyperthermostable CelB from Pyrococcus furiosus in Lactobacillus sp. Appl Microbiol Biotechnol 2012, 96:903-912.

10. Wells JM, Robinson K, Chamberlain LM, Schofield KM, Le Page RW: Lactic acid bacteria as vaccine delivery vehicles. Antonie Van Leeuwenhoek 1996, 70:317-330.

11. Schwarzer M, Repa A, Daniel C, Schabussova I, Hrncir T, Pot B, Stepankova R, Hudcovic T, Pollak A, Tlaskalova-Hogenova H, Wiedermann U, Kozakova H: Neonatal colonization of mice with Lactobacillus plantarum producing the aeroallergen Bet $v 1$ biases towards Th1 and T-regulatory responses upon systemic sensitization. Allergy 2011, 66:368-375.
12. Djordjevic GM, Klaenhammer TR: Inducible gene expression systems in Lactococcus lactis. Mol Biotechnol 1998, 9:127-139.

13. D'Souza R, Pandeya DR, Rahman M, Seo Lee H, Jung JK, Hong ST: Genetic engineering of Lactococcus lactis to produce an amylase inhibitor for development of an anti-diabetes biodrug. New Microbiol 2012, 35:35-42.

14. Jorgensen CM, Vrang A, Madsen SM: Recombinant protein expression in Lactococcus lactis using the P170 expression system. FEMS Microbiol Lett 2014, 351:170-178.

15. Maidin MS, Song AA, Jalilsood T, Sieo CC, Yusoff K, Rahim RA: Construction of a novel inducible expression vector for Lactococcus lactis M4 and Lactobacillus plantarum Pa21. Plasmid 2014, 74:32-38.

16. Kuipers OP, de Ruyter PG, Kleerebezem M, de Vos WM: Controlled overproduction of proteins by lactic acid bacteria. Trends Biotechnol 1997, 15:135-140.

17. Sorvig E, Mathiesen G, Naterstad K, Eijsink VG, Axelsson L: High-level, inducible gene expression in Lactobacillus sakei and Lactobacillus plantarum using versatile expression vectors. Microbiology 2005, 151:2439-2449.

18. Mierau I, Kleerebezem M: 10 years of the nisin-controlled gene expression system (NICE) in Lactococcus lactis. Appl Microbiol Biotechnol 2005, 68:705-717.

19. Pavan S, Hols P, Delcour J, Geoffroy MC, Grangette C, Kleerebezem M, Mercenier A: Adaptation of the nisin-controlled expression system in Lactobacillus plantarum: a tool to study in vivo biological effects. Appl Environ Microbiol 2000, 66:4427-4432.

20. Rossi F, Rudella A, Marzotto M, Dellaglio F: Vector-free cloning of a bacterial endo-1,4-beta-glucanase in Lactobacillus plantarum and its effect on the acidifying activity in silage: use of recombinant cellulolytic Lactobacillus plantarum as silage inoculant. Antonie Van Leeuwenhoek 2001, 80:139-147.

21. Okano K, Zhang Q, Yoshida S, Tanaka T, Ogino C, Fukuda H, Kondo A: D-lactic acid production from cellooligosaccharides and beta-glucan using L-LDH gene-deficient and endoglucanase-secreting Lactobacillus plantarum. Appl Microbiol Biotechnol 2010, 85:643-650.

22. Sasikumar P, Gomathi S, Anbazhagan K, Baby AE, Sangeetha J, Selvam GS: Genetically Engineered Lactobacillus plantarum WCFS1 Constitutively Secreting Heterologous Oxalate Decarboxylase and Degrading Oxalate Under In Vitro. Curr Microbiol 2014, 69:708-715.

23. McCracken A, Turner MS, Giffard P, Hafner LM, Timms P: Analysis of promoter sequences from Lactobacillus and Lactococcus and their activity in several Lactobacillus species. Arch Microbio/ 2000, 173:383-389.

24. McCracken A, Timms P: Efficiency of transcription from promoter sequence variants in Lactobacillus is both strain and context dependent. J Bacteriol 1999, 181:6569-6572.

25. DeVos WM, Simons AFM, Zuivelonderzoek SNIV: Method for preparing proteins using transformed lactic acid bacteria. 1991

26. Heinl S, Spath $\mathrm{K}$, Egger E, Grabherr R: Sequence analysis and characterization of two cryptic plasmids derived from Lactobacillus buchneri CD034. Plasmid 2011, 66:159-168.

27. Spath K, Heinl S, Egger E, Grabherr R: Lactobacillus plantarum and Lactobacillus buchneri as expression systems: evaluation of different origins of replication for the design of suitable shuttle vectors. Mol Biotechnol 2012, 52:40-48.

28. Mattanovich D, Weik R, Thim S, Kramer W, Bayer K, Katinger H: Optimization of recombinant gene expression in Escherichia coli. Ann N Y Acad Sci 1996, 782:182-190

29. Wang T, Ma X, Du G, Chen J: Overview of regulatory strategies and molecular elements in metabolic engineering of bacteria. Mol Biotechnol 2012, 52:300-308.

30. Spath $\mathrm{K}$, Heinl $\mathrm{S}$, Grabherr R: Direct cloning in Lactobacillus plantarum: Electroporation with non-methylated plasmid DNA enhances transformation efficiency and makes shuttle vectors obsolete. Microb Cell Fact 2012, 11:141.

31. Rud I, Jensen PR, Naterstad K, Axelsson L: A synthetic promoter library for constitutive gene expression in Lactobacillus plantarum. Microbiology 2006, 152:1011-1019.

32. Mathiesen G, Sorvig E, Blatny J, Naterstad K, Axelsson L, Eijsink VG: High-level gene expression in Lactobacillus plantarum using a pheromone-regulated bacteriocin promoter. Lett App/ Microbiol 2004, 39:137-143.

33. Grabherr MG, Pontiller J, Mauceli E, Ernst W, Baumann M, Biagi T, Swofford R, Russell P, Zody MC, Di Palma F, Lindblad-Toh K, Grabherr RM: Exploiting nucleotide composition to engineer promoters. PLoS One 2011, 6:e20136. doi:10.1371/journal.pone.0020136. 
34. Shine J, Dalgarno L: Terminal-sequence analysis of bacterial ribosomal RNA. Correlation between the 3 '-terminal-polypyrimidine sequence of 16-S RNA and translational specificity of the ribosome. Eur J Biochem 1975, 57:221-230.

35. De Man JC, Rogosa M, Sharpe ME: A medium for the cultivation of lactobacilli. J Appl Microbiol 1960, 23:130-135.

doi:10.1186/s12934-014-0150-z

Cite this article as: Tauer et al:: Tuning constitutive recombinant gene expression in Lactobacillus plantarum. Microbial Cell Factories 2014 13:150

\section{Submit your next manuscript to BioMed Central} and take full advantage of:

- Convenient online submission

- Thorough peer review

- No space constraints or color figure charges

- Immediate publication on acceptance

- Inclusion in PubMed, CAS, Scopus and Google Scholar

- Research which is freely available for redistribution 